
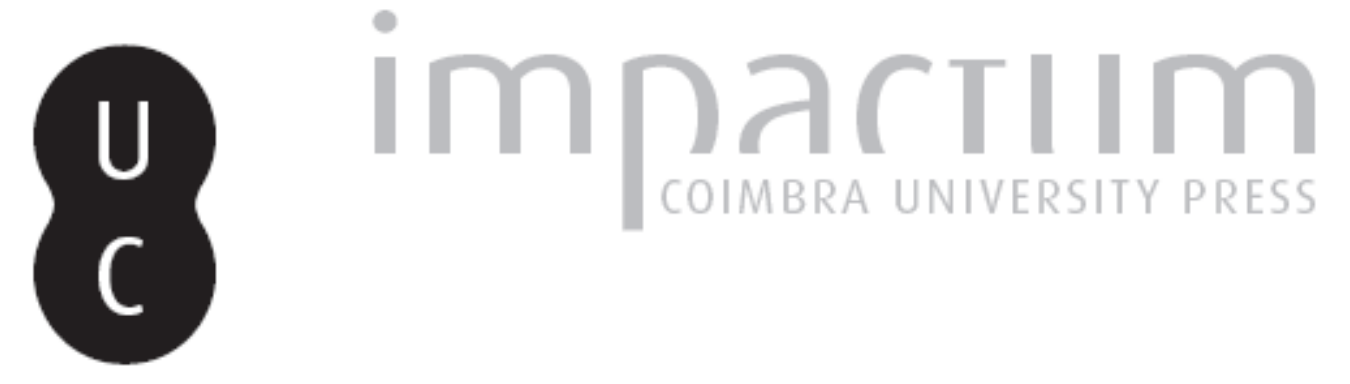

\title{
Malim, Asteriskus und fragezeichen: einige wore zur verteidung und zum lobe von Gregorios N. Bernardakîs
}

\author{
Autor(es): Ingenkamp, Heinz Gerd \\ Publicado por: International Plutarch Society \\ URL \\ persistente: \\ URI:http://hdl.handle.net/10316.2/37609 \\ DOI: \\ DOI:http://dx.doi.org/10.14195/0258-655X_3_5
}

Accessed : $\quad$ 26-Apr-2023 13:07:00

A navegação consulta e descarregamento dos títulos inseridos nas Bibliotecas Digitais UC Digitalis, UC Pombalina e UC Impactum, pressupõem a aceitação plena e sem reservas dos Termos e Condições de Uso destas Bibliotecas Digitais, disponíveis em https://digitalis.uc.pt/pt-pt/termos.

Conforme exposto nos referidos Termos e Condições de Uso, o descarregamento de títulos de acesso restrito requer uma licença válida de autorização devendo o utilizador aceder ao(s) documento(s) a partir de um endereço de IP da instituição detentora da supramencionada licença.

Ao utilizador é apenas permitido o descarregamento para uso pessoal, pelo que o emprego do(s) título(s) descarregado(s) para outro fim, designadamente comercial, carece de autorização do respetivo autor ou editor da obra.

Na medida em que todas as obras da UC Digitalis se encontram protegidas pelo Código do Direito de Autor e Direitos Conexos e demais legislação aplicável, toda a cópia, parcial ou total, deste documento, nos casos em que é legalmente admitida, deverá conter ou fazer-se acompanhar por este aviso.

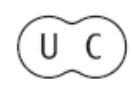




\section{PLOUTARCHOS, n.s.}

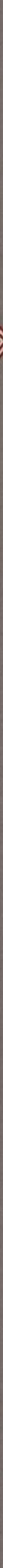

UNIVERSITY OF MÁLAGA (SPAIN)

UtAH STATE UNIVERSity, LOGAN, UTAH (U.S.A.) 


\title{
Malim,Asteriskus und Fragezeichen. Einige Worte zur Verteidung und zum Lobe von Gregorios N. Bernardakis von
}

\author{
Heinz Gerd Ingenkamp \\ Universität Bonn
}

\begin{abstract}
Immediately after its publication, the edition of Plutarch's Moralia by Gregorios Bernardakis (1888-1896) has been strongly criticized, to say the least, by Wilamowitz, who inaugurated the new Teubner-edition (1925ff.). Bernardakis is attacked by Wilamowitz and Pohlenz, who wrote the preface to the new Teubeneriana, because he chose the Parisinus 1956, D, to be the fundament of his Plutarch. The following article tries to prove that $\mathrm{D}$ was a reasonable choice. Four of the proudly presented examples, that Pohlenz hopes will convince the reader of the superiority of the Vw/gata-tradion, will hopefully show that D resp. the Bemardakis-edition may have the more Plutarchan text. The article begins with a sceptical description of the expectations Wilamowitz and most of his contemporaries connected with a Lachmann-style recensio.
\end{abstract}

Wer als Altphilologe über Gregorios Bemardakis sprechen soll, denkt in erster Linie an dessen Hauptwerk, die Ausgabe von Plutarchs Moralia ${ }^{1}$. Es gibt Werke, die einen Philologen gewissermaßen zum Spieler auf der Bühne des philologischen Weltgeschehens machen: Man nimmt weithin Notiz von ihnen, sie erregen die Gemüter, sie haben eine lange Nachwir kung, und all das unabhängig davon, ob die Reaktionen zunächst oder dauerhaft nega tiv oder positiv sind. Bemardakis hat in den vielen Jahrzehnten seines Wirkens Weite- res über Plutarch geschrieben, dazu über Strabo, Sophokles, Epigraphisches, nicht zuletzt auch über Thukydides. Aber es ist seine Plutarchausgabe, die jedermann sofort einfällt, wenn er seinen Namen hört.

Wer von dieser inzwischen mehr als hundertjährigen Ausgabe spricht, hat es sowohl mit dieser selbst als auch mit den Kritiken zu tun, die einige Jahrzehnte nach ihrem Erscheinen zu einer neuen Teubneriana geführt haben. Diese kau sale Verbindung wird von den Herausge bern der neuen Ausgabe selbst herge stellt. Der Anreger der Ausgabe, also ihr geistiger Vater, ist Ulrich von Wilamowitz-Moellendorff. Ihr erster Band

] Plutarchi Chaeronensis Moralia. Recognovit Gregorius N. Bernardakis. Leipzig, $1888 \mathrm{ff}$. 
erschien 1925². Max Pohlenz hat die Einleitung geschrieben und liefert in wenigen Sätzen eine Würdigung der Vorgängerausgabe, die natürlich auch die Kritik enthält, die seiner Meinung nach eine Neuausgabe erforderlich macht. Ich gehe bei meiner Darstellung von dieser und von Wilamowitzens Kritik aus und füge ihr diejenige des noch auf der Bemardakisschen Ausgabe fußenden Herausgebers der ersten Bän de der Loeb-Edition von Plutarchs $M o$ ralia, Frank Cole Babbitt ${ }^{3}$, hinzu.

\section{1. minime operam perdidit}

Pohlenz bescheinigt Bemardakis, er habe eine enorme Leistung erbracht, wenn ich das schlichte und schöne Latein so stillos übersetzen darf. Die von Bernardakis allein betreute Ausgabe erschien in den Jahren von 1888 bis 1896. Zum Vergleich: Die zweite Teuberiana und die Gesamtausgabe der Collection Bude werden nach Erscheinen des je ersten Bandes bis zur Vollständigkeit Jahrzehnte brauchen und von nicht weni gen Editoren betreut werden. Bemardakis hatte sich Ende der siebziger Jahre mit einem kleineren, auch schon bei Teubner erschienenen Buch zur Kritik des Textes von Plutarchs Biographien und seinen Moralia als Herausgeber empfohlen. ${ }^{4}$ Es handelt sich bei Plutarchs Moralia um 78 Schriften, von denen eine, die Quaestio nes convivales, aus 9 „Büchern“ (d.h. Teilen in der Länge von durchschnitt lichen Einzelschriften) besteht. Über die Vorarbeiten, die zur Herstellung einer Ausgabe antiker Texte nötig sind, wird gleich in anderem Zusammenhang noch zu reden sein. Die „enorme“ Leistung der Herausgebers Bemardakis ist eine so gut wie unglaubliche Leistung.

\section{2. fundamentum recensionis non esse iactum $^{5}$}

Diese unglaubliche Leistung wurde dem Gelehrten nicht gerade erleichtert durch eine Kritik, die nicht nur vemich-

2

Plutarchi Moralia, Vol. I. Recensuerunt W. R. Pat onJ et I. Weg e h a upt )*. Praefationem scr. M. Pohlenz. Leipzig 1925. Die Teubneriana setzt die Tradition fort, die die in die Zeit der Bernardakisausgabe zurückgehende Edition der Pythischen Dialoge durch W. R.

Pa t o n fPlutarchi Dialogi tres, Berlin 1893) vertreten hatte.

Plutarchs Moralia in fifteen volumes. With an English translation by Fra nk Col e

Ba bbit t 〈...>, London / Cambridge, Massachusetts 1922ff.

Symbolae criticae etpalaeographicae in Plutarchi Vitas Parallelas et Moralia, Leipzig 1779.

Die PoHLENZsche Formulierung, die ich diesem Abschnitt vorangestellt habe, könnte ein Zitat aus dem ersten Satz dieses Buches sein. Das Philologische Seminar der Universität Bonn besitzt ein Exemplar mit einer handschriftlichen Widmung des Verfassers an Franz Bü-

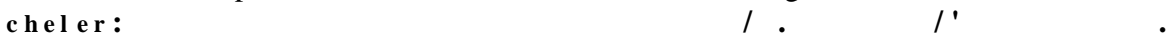

5 U. von Wil a mowitz-Moellendorff, „Commentariolum grammaticum III“, index Scholarum publice et privatim in Academia Georgia Augusta per semestre aestivum a.d. XXIII. m. Aprilis usque ad d. XV. m. Augusti A. MDCCCLXXX1X habendarum, Göttingen 1889, S. 21. 
tend, sondern auch verletzend sein soll te. Für einen Forscher, der mit einer auf mehrere Jahre projektierten Arbeit befaßt ist, ist das die denkbar schlechte ste Bedingung für die Fortsetzung seines Werkes. Die kaum erträgliche Kritik setzte bereits 1889 ein, also ein Jahr, nachdem der erste Band der Ausgabe erschienen war. Was die Lage dann doch halbwegs erträglich machte, war einer seits die gehaltliche Dürftigkeit dieser Kritik und dann gerade auch das dahinter stehende Temperament, das, vielleicht sogar schon bei dem von ihr direkt Betroffenen, also Bernardakis selbst, mehr zu Kopfschütteln als zu wirklicher Empörung Anlaß gegeben haben mag. Derjenige, der sich so unerfreulich expo nierte, war der geistige Vater der späteren Edition, Ulrich von Wilamowitz-Moellendorff, der schon damals auf dem Weg war, zu dem herausragenden Gräzisten zu werden, als den man ihn immer zu schät zen haben wird. Angriffen von einer sol chen Stelle ausgesetzt zu sein, kam einer Herausforderung gleich, die man sich in unseren Zeiten, in denen die Geistes wissenschaft etwas von ihrer Autoritäts frömmigkeit verloren hat, kaum noch vorstellen kann. Worum ging es in der Wilamowitzschen Kritik, jedenfalls in dem Teil, den wir noch am ehesten als sachlich bezeichnen können?
Einige Jahrzehnte vor dem Streit zwischen Bernardakis und Wilamowitz war, im Gefolge der als „Historismus“ bezeichneten kulturellen Revolution, die Textkritik neueren Zuschnitts entstan den. Der Philologe, der sich über den Text eines antiken Autors klarwerden oder diesen edieren will, muß dieser Methode gemäß die gesamte Überliefe rung zunächst ordnen. Da es sich gezeigt hat, daß die manchmal sehr zahlreichen noch vorhandenen Handschriften, die das zu edierende Werk kopiert haben, in der Regel auf eine einzige Urhandschrift, den Archetypos, zurückgehen, wird verlangt, daß man alle diejenigen Handschriften, die nichts als Kopien von noch vorhandenen anderen Hand schriften sind, als solche kenntlich macht und somit aussondert ${ }^{6}$. Die ge samte Textkritik, auch das Aussortieren von Handschriften, beruht auf der psychologischen Gesetzmäßigkeit, daß es unmöglich ist, einen längeren Text fehlerfrei abzuschreiben. Man will also anhand der Fehler Quelle und Kopie voneinander trennen. Am Ende der Prozedur kommt man zu einer relativ kleinen Zahl von Handschriften, die nicht mehr reduzibel ist. Stets dann, wenn diese Handschriften übereinstim men, kann man davon ausgehen, daß

${ }^{6}$ Das allgemein bewunderte und oft neu aufgelegte Werk, das den Geist der recensio teilweise in etwa dem Ton einer zu Kaisers Zeiten abgefaßten Einführung in die Bruchrechnung kon densiert, ist: Pa ul Ma a s, Textkritik, Leipzig (Teubner) 1927. Es findet sich im übrigen in die sem Büchlein, vor allem im Abschnitt über die examinatio, viel Weises (vgl. unten, Anm.28), und es wird, richtig gelesen, den Zugang zum besten Text nicht versperren - Der Vater der Textkritik ist der Altphilologe und Germanist Karl Lachmann (1793-1851). 
man den Text des Archetypos vor sich hat. Nicht immer liegen die Dinge so einfach, aber im Prinzip ist die Methode der Anordnung der Textzeugen, genannt recensio, damit schon skizziert ${ }^{7}$. Der Textkritiker stellt also mit seiner Methode nicht den Originaltext des Autors, sondern den Archetypos wieder her, der durchweg viele Jahrhunderte nach der Niederschrift des Originals entstanden ist. Folglich hat der Archetypos, der seinerseits regelmäßig wieder eine Abschrift von Abschriften ist, Fehler, d.h. er entspricht in vielen Punkten nicht dem Original. Die Fehler des Archtetypos müssen nun verbessert, „emendiert“ werden. Über diesen weiteren Schritt brauchen wir hier nichts zu sagen, weil Wilamowitzens Hauptattacke der mangelnden recensio gilt. Im Ton wesent lich konzilianter, aber in der Sache noch ebenso entschieden, sagt Pohlenz später in seiner Einleitung: veram $\langle\ldots\rangle$ recensionen ne ipse quidem perfecit, worin, liebens würdigerweise, doch auch ein Komplime nt steckt: Sogar Bemardakis, trotz seiner enormen Leistung, hat es noch nicht $\mathrm{zu}$ einer wahren recensio gebracht.

Wenn Wilamowitz in der ersten sei ner Polemiken Bernardakis vorwirft, fundamentum recensionis non esse iactum, daß also durch Bernardakis das Fundament für eine recensio nicht gelegt sei, ist die Ausgabe, mindestens der vorliegende erste Band, gerichtet oder besser hingerichtet. Bernardakis wird sich selbst in der Einleitung zum zweiten Band seiner Einleitung mit Wilamowitzens Kritik auseinanderset zen. Ich meinerseits will mich der Reflexion über die Hoffnungen hinge ben, die ehedem, in den frühen Tagen der Textkritik, mit einer solchen recen sio wohl verbunden worden sind, denn sonst hätte man eine Ausgabe nicht allein deswegen, weil sie, die recensio, unterlassen worden war, auf so entschie dene Weise aburteilen können, wie Wilamowitz das tut. Das Auge für den richtigen, plutarchischen Kodex spielte offenbar nicht nur keine Rolle, sondern muß als eine Art wegzuoperierende Himprotuberanz gegolten haben. Daß die recensio ein wichtiges Mittel zur Herstellung des Textes ist, kann nur purer Unverstand bestreiten; schließlich werde ich selbst meine Argumente zur Verteidigung des Bernardakistextes auf der von Max Treu und anderen vorge legten recensio aufbauen. Daß die recen sio aber andererseits das Auge bei der Wahl der Leitüberlieferung nicht ersetzt, jenes Auge also, über das meines Erachtens Bemardakis verfügt hat, wird wenigstens von der frühen Textkritik und ihrem Propagator Wilamowitz bestritten. Wenn dem meisterlichen Blick die Gestikulation mit dem Handwerkszeug nicht beigegeben ist, so scheint er nichts wert $\mathrm{zu}$ sein. Gegen diesen Allein vertretungsanspruch des Handwerk lichen darf man wohl, wenn er verlet zend wird, seinerseits polemisieren.

\footnotetext{
7 Vgl. Ma as, $\$ 8$.

8 a.a.O. XIV.
} 
Die Einführung der im Grunde trivi aljuristischen Methode der Eliminierung von Kodizes, die für die Textgestaltung nicht nötig sind, wirkte auf die deut schen Philologen der damaligen Zeit wohl wie eine Art Brücke zu den exak ten Wissenschaften. Es war ja die Zeit, in der diese ihren Triumphzug feierten und die Industrialisierung ihrer Bedeu tung und ihrer Nützlichkeit geradezu täg lich neue Beweise lieferten. Endlich war man heraus aus dem Reich der scheinba ren Beliebigkeit und hatte festen Boden unter den Füßen. Es kam hinzu, daß sie auch in zwei anderen Hinsichten zeitge mäß war. Der Historismus ist ein Produkt der Romantik, und wir erleben gegen Ende des 19. Jahrhunderts eine Art zweite Romantik, die wir Spätromantik nennen. „Zurückgehen zu den Quellen“ war eine Devise, die man nicht lernen mußte, son dern die in der Luft lag, und die histori sche Methode stellte den Ariadnefaden dazu zur Verfügung. Natürlich war klar, daß der Archetypos eine Handschrift besonderer Art war, wie Blut ein ganz besonderer Saft ist. Und ein Drittes trat hinzu. Der Archetypos ist unserer direk ten Sinneswahmehmung meistens entzo gen, aber er regiert den Rest der Überlie ferung. Er ist ein pharaonenhafter Po tentat. Wir einfachen Menschen erkennen ihn durch die nächstfolgenden Stufen. Die Stammbäume von Kodizes, die man in den Ausgaben sieht, erinnern an militäri sche Rangfolgen. Oben eine schmale, jen seitige Spitze, unten verbreitert sich das Bild bald zum Kanonenfutter. Zu tun hat der vornehme Editor mit den Feldmarschällen und Generälen.

Die Naivität des - in der Kritik an Bernardakis indirekt zutage tretenden Wilamowitzschen Methodenoptimismus angesichts der unübersehbaren Möglich keiten von erstaunlicherweise gelegent lich auch unentdeckbaren Fehlem, liai sons dangereuses und Eigenmäch tigkeiten bei der sich über viele, sehr viele Jahrhunderte hinziehenden handschrift lichen Kopierarbeit - ohne das wachsame Auge eines Copyright im Hintergrund kann nur verstanden werden, wenn man ihn, diesen Optimismus, auf jene Epochen vornehmlich der deutschen Geschichte bezieht, in denen er konse quent vertreten wurde. Mit der späteren, die strenge Textkritik entscheidend schwächenden Parole Giorgio Pasqualis recentiores non deteriores (jüngere Handschriften sind nicht unbedingt schlechter) stürzt ein Teil der hierarchisierten Textkritik ein ${ }^{9}$. Auf einmal konnte ein Oberst, ein Leutnant, ohne viel Umstände als tüchtiger anerkannt sein als ein General. Das wußte man beim Militär schon immer, auch die Textkritiker hatten es gemerkt, aber es zur Parole zu machen, bedeutete doch eine Entwicklung.

Doch auch ohne eine solche förderli che Entwicklung war von vornherein offenkundig, wo die Grenzen der oft 
apodiktisch gegen andere Formen der Vorlagenwahl vertretenen recensio die ses Typs lagen. Man hatte der Philologie auf einem ihrer Gebiete eine Art Zuverlässigkeit gesichert, das schien festzustehen, aber natürlich war diese Zuverlässigkeit anderer Art als diejenige von physikalischen Errungenschaften. Konnte der Physiker experimentell nachweisen, daß seine Theorie stimmt, so mußte sich der Textkritiker damit begnü gen, daß er seine Fachkollegen zwingen konnte, ihm Recht zu geben, und das auch nur dann, wenn die Fachkollegen sich der eingeforderten Methode unter warfen. Der Horizont der recensio, und vor allem schon der Druck, eine der ein geforderten Methode konforme recensio als so entscheidend empfinden zu müs sen, daß bei Nichterfüllung das ganze Werk hinfällig wird, ist nichts anderes als der bloße consensus omnium bonorum (philologorum), etwas weniger verwa schen formuliert, der Parteigänger in methodicis, jedenfalls nicht der antike Text, den man ja nicht mehr hat. Es ist klar und zu akzeptieren, daß bei einem solchen Kriterientyp die gegenseitige Bloßstellung, der Nachweis der Inkompetenz des anderen, kurz und häß lich gesagt: seine Erledigung schlichtweg zum Handwerk gehört. Das Gefährliche bei der Textkritik alten Typs bestand und besteht darin, daß diese an sich legitime ${ }^{10}$ „Erledigung“ auf dem Hintergrund eines unbegründeten Exaktheitswahns erfolgt.

Was hat Bernardakis denn nun getan?

codicem Parisinum $D<\ldots>$ tam quam sincerum testem secutus esi ${ }^{1}$.

Zunächst einmal hat er weder der recensio, wie Wilamowitz sie wollte, den Kampf angesagt, noch hat er sie ein fach ignoriert. Er sagt nämlich, bevor er mit der Beschreibung der handschrift lichen Textgrundlagen des Plutarchtextes beginnt, daß er auf den Vor arbeiten von Max Treu fußt. Max Treu aber hatte unschätzbare Arbeit auf dem Gebiet der recensio geleistet. Wilamo witz stellt die Sache so dar, als habe Bernardakis die Leistung Treus herabge setzt, und fordert Treu in seinem Commentariolum grammaticum pathe tisch auf, sich nicht von Bernardakis einschüchtern zu lassen, der seinen ver dienten Ruhm geschmälert habe. Bernardakis ist über die Anschuldigung empört und weist sie als Verleumdung zurück (.Moralia, Band II, Seite Xlf.). In Wirklichkeit nutzt er die Resultate Treus, und er kritisiert sie, wo er es für angebracht hält. Er setzt sich also offen sichtlich nicht über das Postulat der ein geforderten recensio hinweg, sondern verwendet das diesbezügliche Material,

Ich kann verstehen, wenn man mir das Urteil „legitim“ nicht durchgehen lassen will. Aber ich tröste mich mit dem Schluß von Horazens Ars Poetica (vv. 453ff), der mir hel fen wird, meine Rechtfertigung in Angriff zu nehmen. 
das zu seiner Zeit vorliegt, um dann sei

nen eigenen Weg zu gehen. Das war offenbar die eigentlich Sünde ${ }^{12}$.

Sie bestand, genauer gesagt, darin, daß er, wie schon sein Vorgänger, Rudolf Hercher, dessen Moralia-Ausgabe von 1872 aber über den ersten Band nicht herausgekommen war, einen bestimmten vorhandenen Kodex zum besten Textzeugen erklärte ${ }^{13}$. Sehr wohl wissend, daß die komplizierte Überliefe rungslage im Falle der Moralia Plutarchs einen und denselben Kodex für die eine Schrift einen zuverlässigen Zeugen sein läßt, für eine andere dagegen nicht, hatte Bernardakis den Kodex D, den Parisinus
12

Die Intransigenz, mit der Wil amowit z alternative Wege der Textkonstitution vom Tisch zu fegen beabsichtigt, hängt natürlich mit dem irrationalen Sicherheitsgefühl des Vertreters einer „modernen“ Methode zusammen. Es kommt im Zusammenhang damit zu Anwürfen, deren Eigenart allerdings damit nicht zusammenhängt. So verwendet er in seiner ersten Attacke die Formulierung mercennaria opera im Hinblick auf die Arbeit von Bernardakis (Comment, gramm. III, p.21). Der Angegriffene reagiert in der praefatio zum 2. Band eini germaßen erstaunt. Darauf nun wiederum antwortet Wilamowitz mit der von der Argumentstruktur her gesehen kindergartenreifen Anmerkung (Hermes 1890, p. 207, Anm. $2=K l$ Sehr. [Uirich von Wilamowitz-Moellendorff, Kleine Schriften, hg. von den Akademien zu Berlin und Göttingen, Berlin, 1969] III, 128, Anm.): „Befremden könnte mich, daß Herr Bernardakis sich so gebärdet, als wisse er nicht, was mercennaria opera bedeute. Er hat wohl von der Antwort keine Kunde, die ich seinem Verleger erteilt habe, als dieser mir seinen danach fragenden Brief übersandte. Diese Antwort würde ihm freilich die Gelegenheit zu schönen Deklamationen geraubt haben.“ Ebenfalls an den Kindergarten, Abteilung Knaben, erinnert der Schluß der Anmerkung auf S. 199 / 120 desselben Aufsatzes. Wil amowitz trägt einige sprachliche Schnitzer vor, die er selbst gemacht hat, und fährt fort: „Auch diese Übereilungen stammen in Wahrheit aus derselben Unart meines Wesens wie die Druckfehler. Ich weiß das aber wohl, ich schäme mich dessen und bemühe mich, es bes ser zu machen: aber vor mir schäme ich mich: vor denen, die mir solche Fehler triumphie rend Vorhalten, brauche ich mich nicht zu schämen." Sechs Jahre später ist der Zorn noch nicht verraucht. Am Schluß der Rezension von W. R. Patons Ausgabe von Plutarchs De cupididate divitiarum (Gött. Gelehrte Anz., 1896, p. $340=K l$. Sehr. III 177) meint er, das Folgende von sich geben zu sollen: „Blaß hat sich von Bernardakis den fünften Band der Moralia widmen lassen amicitiae ergo; das würde ich auf griechisch etwa übersetzen

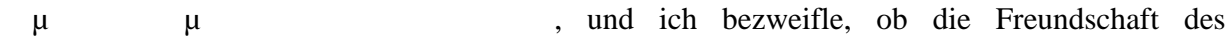
Timarchos für Demosthenes dauernd eine reine Freude gewesen ist." Das ist nicht Kindergarten, sondern (trotz des netten Witzes mit Blass und Demosthenes) Boxring, und dort der Schlag unter die Gürtellinie. Wil amowit z war wohl stolz darauf, denn er beschließt seine Ausführungen mit diesem flourish.

13

Plutarchi Chaeronensis Moralia, ex recensione Rudolfi Hercheri, vol. I, Leipzig, 1872. Wil amowitz, Hermes 1890, 200, wirft Bernardakis übrigens ,sclavische Abhängigkeit“ von Hercher vor. Damit hat sich Bernardakis selbst in der praefatio zu Band IV erfolg reich auseinandergesetzt (p. X ff.). Pohı enz sagt zum „,Fall D“ schlicht „২..> quod [B.] cum Herchero codicem Paris. D <...> tanquam sincerum testem secutus est“" 
1956 aus dem 11. bis 12. Jahrhundert, für insgesamt den besten Textzeugen und zum certissimus dux (I, XIX) ${ }^{14}$ bei der Konstitution des Textes erklärt. Für die Ausgabe bedeutet das, daß er den Lesarten dieses Kodex vorzugsweise folgt. Nun enthält der Kodex D aber nur gut ein Viertel der Moralia, wenn man nach Titeln rechnet. Dem Umfang nach ist, was er bietet, weniger. $\mathrm{Zu}$ der Masse der übrigen Schriften mußte Bemardakis andere Zeugen heranziehen. Aber es war die Wahl von D, mit der er gewisserma Ben seine Karten auf den Tisch gelegt hatte, und so beschränke ich mich folgen den auf den „Fall D“.

Wir können uns ein Urteil über sein Vorgehen erlauben, wenn wir uns kurz vergewissern, um was für einen Kodex es sich im Falle von D handelt. Um in dem polemischen Umfeld, in dem seit Wilamowitz immer über die BernardakisAusgabe geredet worden ist, möglichst sicher zu gehen, wiederhole ich, was Max
Pohlenz dazu sagt. Pohlenz bezeichnet Wilamowitz nicht nur als patronus der neuen Edition, sondern auch als Mitleser der Korrekturen ${ }^{15}$. Also wird man davon ausgehen können, daß Wilamowitz Pohlenzens Einleitung kannte und das hier Vorzutragende im Jahre 1925 billigte $^{16}$.

Pohlenz behandelt den Kodex D in seinem den $\dot{\eta} \theta 1 \kappa \dot{\alpha}$ (Bücher 1-21) gewid meten Abschnitt - natürlich, denn D hat so gut wie nur Schriften aus diesem Werkteil; dazu kommen lediglich De E apud Delphos sowie ein Stück von De defectu oraculorum. Nach Pohlenzens Darstellung gehen alle unsere Kodizes, die die $\dot{\eta} \theta 1 \kappa \dot{\alpha}$ überliefern, also auch $\mathrm{D}$, auf einen gemeinsamen Archetypos zurück. D bildet zusammen mit einigen anderen Handschriften eine Familie, d.h. diese Untergruppe geht auf einen eigenen sogenannten Hyparchetypos, $\Sigma$, zurück $^{17}$. Nun hat aber D, zusammen mit den Kodizes einer anderen Familie, $\Theta$ genannt, oft einen anderen Text als die übrigen Kodizes, auch als die der eige-

14 Die praefationes der BERNARDAKisausgabe zitiere ich kurz mit römischer Band- und Seitenzahl.

15 a.a.O. p. VI.

16 Meine folgenden Überlegungen beruhen nicht auf eigenen Kollationen von Kodizes, sondern auf dem, was die Herausgeber der Moralia über die Ergebnisse solcher Kollationen in den Einleitungen $\mathrm{zu}$ ihren Editionen mitteilen. Ich denke, die Beschränkung auf das genannte Material ist gerechtfertigt. In den Einleitungen werden die Kämpfe zwar nicht bis zu Ende ausgefochten, wohl aber in ihrer Stoßrichtung cha rakterisiert und der erwartete Sieg wird oft an dem einen oder anderen selbstbewußt prä sentierten Fallbeispiel vorweg ausgekostet. Hier hat man also die Schlacht in nuce, und man wird von dem hier Zur-Verfügung-Gestellten auf den Rest schließen können. Die Verfasser wollen jedenfalls, daß man es tut.

17 a.a.O. p. XXX. 
nen Familie, und Pohlenz fragt sich, ob das an willkürlichen Änderungen seitens des gelehrten Schreibers von D oder aber an dem Umstand liegt, daß D hier und da auf eine Überlieferung zurückkgreift, die wir sonst nicht mehr kennen. Willkürliche Eingriffe des Schreibers schließt er nicht nur nicht aus, sondern warnt energisch vor ihnen, aber er glaubt doch, daß manche Lesart, die D allein bietet, und anderes, was D samt der Familie $\Theta$ aufweist, auf eine Tradition zurückgeht, die uns sonst nicht mehr vorliegt, und die er nennt. Im Zuge dieser Untersuchung ergibt sich, was Bernardakis noch nicht wissen konnte, was ihn aber, wenn er es gewußt hätte, sehr gefreut hätte, nämlich: daß $\mathrm{D}$ nach Meinung der Editoren der neuen Teubneriana auf einer gelehrten Ausga be beruht, die lange vor dem Einsetzen der uns sonst vorliegenden Überliefe rung veranstaltet worden ist. Pohlenz berichtet, daß ihm ein junger Orientalist, Paul Keseling, eine syrische Überset zung von Plutarchs Schrift Пврi/ $\dot{\alpha} о \rho \gamma \eta-$ oi $\alpha$ aus dem 6. Jahrhundert vorgelegt hat, die häufig ausschließlich mit der Familie übereinstimme. Die diese Familie begründende Ausgabe ist „nur wenige Jahrhunderte“ (Pohlenz) nach Plutarch zustandegekommen, anders als die sonstige Überlieferung, die auf hochbyzantinische Zeiten zurückgeht. Deren frühester Textzeuge ist ein Palimpsest aus dem 10. Jahrhundert, den Bernardakis zur Zeit seiner Edition übri gens auch noch nicht kennen konnte ${ }^{18}$.

Das in Band I von Pohlenz Mitgeteilte ist in den späteren Einleitungen nicht modifiziert worden. Auch für einige spä tere Bände verfaßt Pohlenz die Einleitun gen; weder in seiner Einleitung zu Band III (1929), noch in denjenigen zu Band V (1960) und VI (1959) ändert sich etwas in der uns interessierenden Sache. Wo D nicht Zeuge sein kann, wohl aber die $\Theta$ Tradition bzw. Teile davon vorliegen, hätte Gelegenheit zu einer Wiederaufnah me des Themas bestanden.

Nun muß man bedenken, daß die grie chische Welt im 4., 5. und 6. Jahrhundert, also zu der Zeit, als die Grundlage für Teile des Textes von D geschaffen wurde, noch in besonders enger Verbindung mit der Antike stand. Keine saecula obscura hatten diese Verbindung unterbrochen. $\mathrm{Zu}$ Anfang des 5. Jahrhundert starb Johannes Chrysostomos; es folgten die Konzilien von Ephesos und Chalkedon, 444 starb der Kirchenvater Kyrillos von Alexandrien, die Mathematikerin Hypatia gehört in dies Jahrhundert, der Lexikograph Hesych und ebenso der gleichnamige Verfasser des Ovo $\alpha$ to $\lambda$ óyo, eines von der Suda ver wendeten literaturgeschichtlichen Lexi kons. Im 6. Jahrhundert lebten die Historiker Prokop, Agathias und Malalas. Plutarch war (Mittel-)Platoniker gewesen; der große Neuplatoniker Proklos lebte im 5. Jahrhundert; im 6. Jahrhundert folgte Simplikios. Zunächst einmal wird man
18 a.a.O. p. XXXIII-XXXVII. 
eine Überlieferung, die auf Plutarch kultu rell so nahestehende Zeiten zurückgeht, nicht ohne gewichtige Gegengründe unter schätzen dürfen. Dann aber wird man auch die Eingriffe in korrupte Textstellen, die damalige Gelehrte vomahmen, kaum des halb allein von der Hand weisen, weil sie aus so alter Zeit stammen. Man war damals zwar noch nicht mit moderner Textkritik vertraut, aber vielleicht war die Zugehörigkeit zur selben Kultur, in der Plutarch selbst noch gestanden hatte, und zur selben Sprachgemeinschaft nicht hinderlich, wenn man eine Textstelle zu sanieren hatte.

Der Griff zum Kodes D, der von einer in solche Zeiten zurückgehenden Tradition profitiert, kann also für die Gelehrten, die D eine so beachtliche Herkunft zuschreiben, so ganz unvernünf tig nicht gewesen sein. Pohlenz macht aber gerade diesen Griff zu einem der Gründe, die zur Notwendigkeit einer neuen Ausgabe führen. Die Argumenta tion, mit der er seinem Leser eventuelle Sympathien für D ausreden will, ist erstaunlich gewunden. Er schreibt: „Es ist natürlich jedermann klar, daß ein Gelehrter jener Zeit [sc. vor dem Zustandekommen der syrischen Überset zung], der daranging, den Plutarchtext zu verbessern, nicht allein von der uns zur Verfügung stehenden Überlieferung abge hangen hat. Wenn wir also einerseits allenthalben (ubique) sehen, daß dieser Gelehrte mit größter Willkür in den Text eingegriffen hat und wir mit größtem Mißtrauen und Vorsicht Vorgehen müs sen, so steht andererseits doch fest, daß dieser Plutarchtext auch genuine und aus alter Tradition stammende Lesarten bewahrt hat. Und es gibt eine Menge von Stellen, wo man zweifeln kann, ob die Lesart von oder die der Vulgata [also der heute in erster Linie berücksichtigten Tradition] vorzuziehen ist" (XXXV). Wenn ein Rechtsanwalt das einem Richter vorträgt, wird er seiner Sache nicht sehr genützt haben. Abgesehen davon, daß das Argument auf der Grenze der Wider sprüchlichkeit laviert, ist die Unterstellung, die Abweichungen von der Vulgata seien auf „größte Willkür“ zurückzuführen, eine petitio principii.

Es kommt noch etwas hinzu. Auch die codices Planudei (Sigle: П, 13./14.Jh) haben haud paucis locis - anders als die zur „Vulgata“ erklärte Kodexmasse - den vor dem 6. Jh. anzusetzenden Text, auf dem die syrische Übersetzung beruht (p. XXXVII). Planudes ist auch kein Rezensionsfundamentsleger im Sinne der frühen Textkritik ${ }^{19}$, aber er war ein gebil deter Landsmann Plutarchs, der seinen Plutarchtext vielleicht besser verstand, als man ihn preußischerseits über „Methode“

Wenn Wilamowitz das erregt-umgangssprachliche formulierte Urteil „Ich bin mit Herrn Bernardakis fertig“ veröffentlicht (Hermes, 1890, $207=K l$. Sehr. III 128), soll das wohl so wirken wie fürstlicher Gnadenentzug bei gleichzeitigem Ausschluß aus der Handwerkskammer. 
$\mathrm{zu}$ fassen bekommt. Mit der gewohnten schlichten Offenheit, die sich nur bei dem eben zitierten Satz beurlaubt hat, heißt es dann bei Pohlenz: et $\Lambda$ et [die Korrek tur eines Moskauer Kodex, die öfter mit den Planudei zusammengeht] $\Pi \Theta$ genuina praebere possunt.

Nach diesen allgemeinen Überlegun gen sollte man die eine oder andere Einzelstelle heranziehen, wenn es darum geht, die Gültigkeit der Teubneriana und das angebliche Veraltetsein der Bemardakisausgabe $\mathrm{zu}$ kontrollieren. Ich wähle dazu die folgenden Stellen aus Pohlenzens Einleitung, die gewisserma ßen zu seinen Paradebeipielen gehören.

1. Zuerst greife ich $\mathrm{zu}$ der relativ

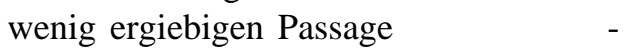

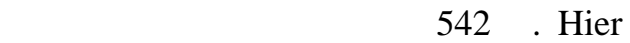
will Pohlenz zeigen, daß D willkürlich emendiert (pp. XXXIV, XXXV unten). Plutarch erzählt von einem Sprecher, der in sein Eigenlob geschickt das Lob seiner Zuhörer einflicht: So werde das Eigenlob erträglich. Bernardakis gibt nach D den folgenden Text:

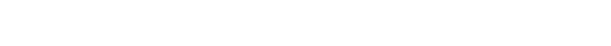

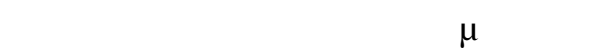

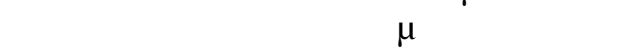
<...> (Denn ohne daß der Zuhörer, der sich über das über ihn Gesagte freut, etwas merkt, schlüpft er [der Sprecher] in das Selbstlob mit hinein). Das kursiv Gedruckte ist die inkriminierte Interpo lation. Die Vulgata hat:

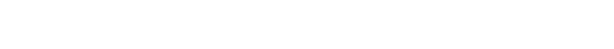

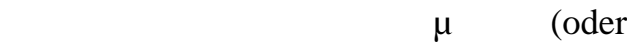

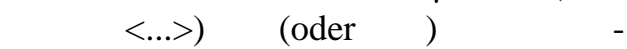

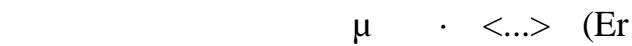
fällt so dem in das Eigenlob miteintauchenden Zuhörer nicht [unangenehm] auf, weil er das [Eigenlob, oder, wenn tó richtig ist: den Ausspruch] des

Sprechenden erfreut annimmt), тoî

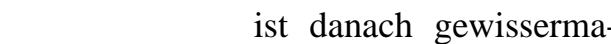
Ben die „Eigenlobsituation“, die man an und für sich nicht mag, aber die man hier akzeptiert, weil man auch für sich selbst einen Platz darin findet.

Die Version der Vulgata des ersten Satzabschnittes ist die facilior, d.h. nach den Regeln der Textkritik eher nicht die ursprüngliche Version. Im Deutschen läßt sich diese Simplizität gut demonstrie ren, indem man, wie oben geschehen, bei der Übersetzung eine attributive Partizipialkonstruktion verwendet. Psycholo gisch ist das sehr einfach: Ich akzeptiere dein Selbstlob, wenn du mich gleichzeitig mitlobst; andernfalls nämlich würde ich von deinem Selbstlob abgestoßen. Der Rest des Satzes ist stilistisch holprig, aber grammatisch korrekt.

Dagegen ist die Version des ersten Satzteils, wie D ihn bietet, komplizier

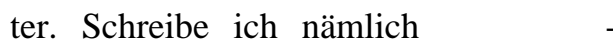
Evo, dann heißt der erste Teil des Satzes, wie gesagt: „Er bleibt dem Zuhörer verborgen, indem er [der Sprecher] in das Selbstlob mitein- 
taucht.“ In was für ein Selbstlob? In das, das ihm selbst gilt? In das braucht er nicht $\mathrm{m}$ /Yhineinzutauchen. In das Selbstlob, das ihm, dem Sprecher und dem Zuhörer gilt? Auch in dieses braucht er nicht mz/einzutauchen, denn er spricht ja aus der Position dessen, der schon ganz in das Selbstlob eingetaucht ist. Er lobt sich ja selbst, тơ̈ [possessiv

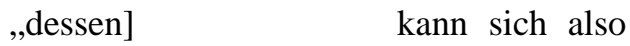
nur auf das Lob des Hörers, des $\dot{\alpha} \kappa \rho о \alpha \tau \dot{~}$ beziehen, und es verlangt eine Assozia tionsbereitschaft, wie man sie bei Tacitus gelegentlich aufzubringen hat, um dahin terzukommen. Die Besitzangabe hat keine Stütze in einem Verbalbegriff: Das ist ihr Problem. Die Vorstellung $\dot{\alpha} \kappa \rho о \alpha \tau \dot{~}$ ist andererseits für den Autor stark genug, um die folgende Besitzanzeige direkt auf ihn zu beziehen.

Gehen wir einmal davon aus, daß D den ursprünglichen Text hat: Wie kommt es dann zum Vulgata-Text? Nach einem ersten Eingriff in den Text, durch

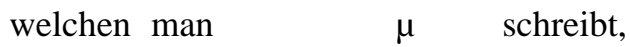
ergibt sich auch ein guter Sinn, wenn

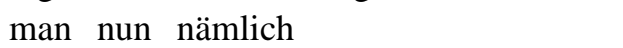

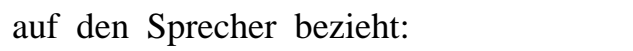

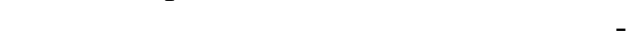

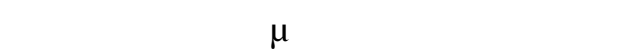

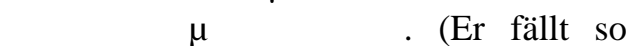
dem in das Selbstlob [des Sprechers] miteintauchenden Hörer nicht auf, der sich über das über ihn, den Hörer, Gesagte freut.). Nun braucht nur noch in einer Vorlage $\lambda \varepsilon \gamma o$ ह่vor zu $\delta \varepsilon \chi 0$ ह่vor ver schrieben worden zu sein (es dürfte sich um einen von einer Minuskelvorlage ver ursachten Sehfehler handeln, aber auch ein Majuskelfehler ist nicht ausgeschlos sen [ $\Lambda$ EГOMENOIL - EXOMENOIL) eine näherliegende Verschreibung ist schwer vorstellbar - und schon sind die Verbesserer am Werk, die dem Text weiter zusetzen, ihn kommentierend ausgestal ten, bis der Vulgata-Text komplett ist.

Es ergibt sich aus Pohlenzens Prunkstelle also nicht, daß der Text in D unbedingt interpoliert sein muß. Der Text der Vulgata kann interpoliert sein und D kann den originalen Text haben. Natürlich behaupte ich das nicht. Und überhaupt macht eine Schwalbe noch keinen Som mer (aber ich wette, daß es noch einige andere flugbereite Vögel dieser freund lichen Gattung gibt). In diesen Dingen hal te ich es lieber mit der unseren Möglich keiten angemesseneren Zurückhaltung von Bemardakis, über die gleich noch zu sprechen sein wird. Es ging mir nur um die Haltbarkeit der Argumentationen, mit deren Hilfe die Wahl des Kodex D zur Textgrundlage bestritten und Bemardakis zur Strecke gebracht werden sollte.

Die neue, unter den Auspizien Wilamowitzens entstandene Teubneriana selbst hat einen Text, der nun seiner seits in den Vulgatatext, der ja zur Not haltbar ist (man könnte an die Hinzu

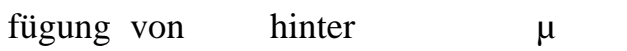
denken, um ihn zu entkrampfen), wak-

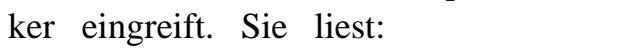

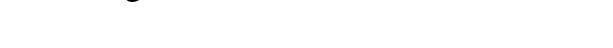

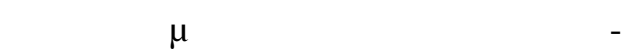
$\omega \pi \rho \circ \delta \delta \varepsilon \chi \dot{0} \varepsilon v o:\langle\ldots\rangle$ : „Denn so nimmt der Hörer unversehens in das ihm 
geltende Lob das darin miteingetauchte Lob des Sprechenden freudig auf", Das könnte man als eine leserfreundliche Glättung ansehen, insofern als das Muster einer lectio facilis. Aber sie beruht ja auf bißfester Methode.

2. Interessanter dürfte ein weiteres Beispiel aus der Pohlenzschen praefatio (p. XXXI) sein, das Пврi $\dot{\alpha} о \rho \gamma \eta \sigma i \alpha$ 453 E betrifft. Pohlenz bringt den Fall als Beispiel für ein Versehen bei der Abschrift aus einem Majuskelkodex. Die Lesart in D könnte m.E. hier die ur sprüngliche sein. Der Text des Pohlenz

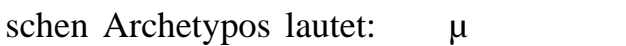

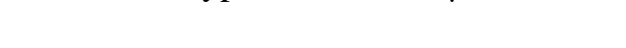

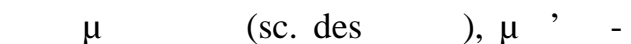

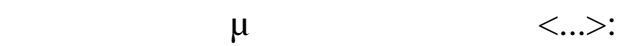
„Denn die Wirkkraft des $\lambda$ óyo gleicht nicht Medikamenten, sondern gesunder Nahrung, die zusammen mit Edelmut eine gute Gesamtdisposition herstellt". Statt des merkwürdigen EYTENEIA $\Sigma$ habe ursprünglich EYTONIA $\Sigma$ im Text gestanden, sagt Pohlenz, womit er sicher überzeugt, wenn seine Prämisse (beste hend im Ergebnis seiner recensio) rich tig ist. Eine andere Lesart, neben dem

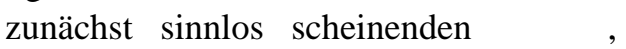
ist $\varepsilon \dot{v} \varepsilon v \varepsilon i \alpha$. Das kann natürlich leicht

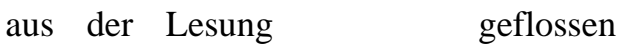
sein. Nun hat D (neben einem weiteren Kodex) عن่vor $\alpha$, was für Pohlenz natür lich eine nachträgliche Konjektur ist.

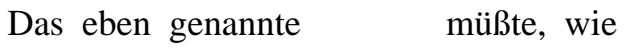
man jetzt sieht, natürlich nicht als Zeugnis für $\varepsilon \dot{v} \varepsilon v \varepsilon i \alpha$, sondern für

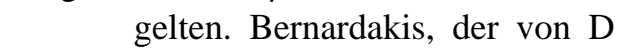
ausgeht, schreibt ن́yıi $\alpha$, setzt also wohl einen Minuskelfehler voraus ( $\varepsilon \dot{v}$

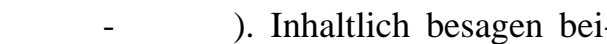
de Konjekturen, die von Pohlenz und die von Bernardakis, dasselbe. Der (in D also nur leicht entstellte) Bemardakistext ist inhaltlich vorzuziehen. Plutarch sagt, die Wirkung des $\lambda$ óyo gleiche nicht etwa medizinischen Heilmitteln, sondern gesunder Nahrung, indem sie

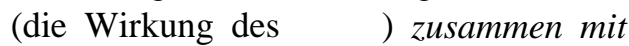
der schon vorzufindenden Gesundheit eine gute Gesamtdisposition bewirke. Gesundheit, heißt das, wird von gesun der Nahrung in ihrer Eigenschaft als ge sunde Nahrung bewirkt: darauf kommt es im Gedanken nicht sehr an. Als gesunde Nahrung, die nicht nur in Krankheitsfällen und für eine besondere Situation nützlich ist, sondern den Körper dauerhaft aufbaut und stabili siert, bewirkt sie aber eine gute Gesamt disposition ( $\left.\dot{\varepsilon} \xi_{1}\right)$. Der Bemardakissche Text hält den Gedanken an die Gesundheit bei seinem zweiten Auftre ten vernünftigerweise als bloße Erinner ung, insofern als Begleitung und zwei rangig, zurück, während die Beziehung „Nahrung $\longrightarrow$ Gesamtdisposition“ her vorgehoben wird. Und in der Junktur $\varepsilon \theta^{\prime} \dot{v} \gamma \imath \varepsilon \dot{ } \alpha \operatorname{mu}$ das $\varepsilon \theta^{\prime}$ betont wer

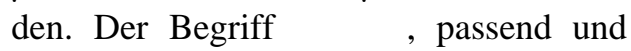
gut wie er ist, würde diesen Fluß leicht stören, weil er zu viel Aufmerksamkeit für eine Nebensache beansprucht. Ist

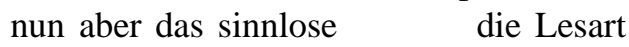
des eigentlichen Archetypos, also der Quelle der -Tradition, so ist natürlich auch nicht schwer zu erklären, wie es durch einen Lesefehler zu der Lesart $\varepsilon \dot{v}$

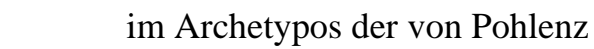


vertretenen recensio gekommen ist. Hier mag man an einen Hörfehler beim inne ren Diktat denken (ewnias - ewjenias). Aber es kann auch eine gelehrte Konjek

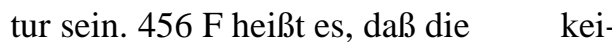

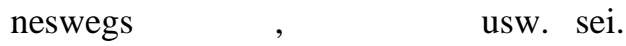
Der Schreiber, der den Traktat kannte, kann bedacht haben, daß es gerade der $\lambda$ óyo ist, der in einem Fall, wo man in Zorn geraten könnte, stattdessen für عن่-

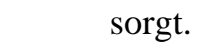

3. Ein drittes Beispiel zeigt, daß Bernardakis D nicht blind folgt, sondern abwägt. Plutarch sagt, Diogenes habe

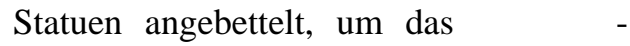
veıv zu üben; ,wir aber wollen uns zunächst am Unscheinbaren und Klei nen üben, nicht ja zu sagen, wenn es nicht gerechtfertigt ist, $\dot{\omega}$ af $\varepsilon i \zeta o \sigma v v$

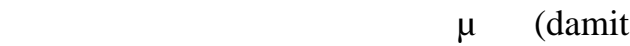
wir größerem Scheitern Vorbeugen kön nen).“ Es handelt sich um die Stelle

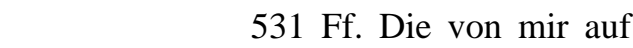
griechisch zitierten Wörter sind nach Pohlenz eine Interpolation der Hand schriftengruppe $\mathrm{M}^{2} \Pi \Theta \mathrm{J} \mathrm{K}$, und damit möglicherweise ein Eingriff von Planudes selbst. Bernardakis folgt dieser Gruppe und gibt nur im Apparat mit gewohnter Zurückhaltung an, daß man vielleicht dem ,besten“ Kodex D folgen sollte, der diese Worte und eines davor ausläßt. Aber er folgt D eben nicht. Der sich bei Bernardakis ergebende Text ist gut Plutarchisch. Diogenes bettelt

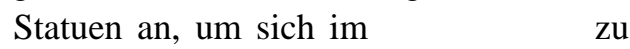
üben: d.h. im Hinnehmen von einem „Nein“ seitens der Angebettelten. Ebenso sollen wir uns an Kleinem üben, um Situationen, in denen wir aus fal scher Scham „Ja“ zu sagen bereit sind, vorzubeugen. Nun ist $\dot{\alpha} \pi \dot{\tau} \varepsilon v \xi_{1}$ in die sem Zusammenhang natürlich etymolo

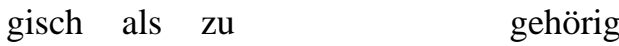
gedacht. Nur heißt $\dot{\alpha} \tau о \tau u \gamma \chi \dot{\alpha} v \varepsilon ı$ bei Plutarch nun in dem einen der beiden Fälle: „ein Nein einstecken“ und beim zweiten Mal „bei der falschen Gelegen heit Ja sagen“. Ist das möglich? Nun,

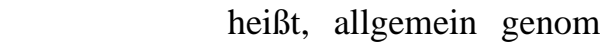
men, scheitern. In beiden Fällen droht ein Scheitern: Diogenes bekommt von den Statuen nichts; wir sagen vielleicht Ja, wenn wir es nicht sollten, wir geben vielleicht, wo wir es eigentlich nicht sollten und möchten. Im ersten Fall ist $\dot{\alpha} \pi 0 \tau \cup \gamma \chi \dot{\alpha} v \varepsilon 1 v$ in jeder Hinsicht pas send: Sowohl mit seiner allgemeinen Bedeutung ,scheitern“, als auch mit der speziellen Bedeutung ,etwas nicht be kommen“. Gesetzt, der von Paton und Pohlenz angezweifelte Text ist eine Konjektur: Dann ist sie genial, denn sie arbeitet mit den bei Plutarch beliebten „puns“, „Kalauern“, die ein Zitat, eine Parallele u.ä., leicht verschieben, um den Leser zu amüsieren. Hier steckt der Witz in der überraschenden Verwendung eines Wortes in einer auf den ersten Blick verwirrenden, auf den zweiten Blick belehrenden Weise. Denn auch dann, wenn wir, ohne es zu wollen, Ja sagen, erreichen wir etwas nicht: Näm lich bei unseren Prinzipien oder auch nur bei unseren Interessen zu bleiben. Daß ein solcher Jokus Plutarch zuzutrauen ist, wird mir jeder seiner Leser bestätigen. Dagegen ist die Konjektur von Paton, die 
Pohlenz in den Himmel hebt (,egregio supplemento emendavit"), simpel. Wie $\dot{\alpha} \pi \dot{\tau} \tau \varepsilon v \xi_{1}$ sich auf das vorhergehende $\dot{\alpha} \pi$ то $ү \chi \dot{\alpha} v \varepsilon ı v$ beruft, so nimmt Paton aus den sofort folgenden Worten das Mittel zur Abhilfe. Was herauskommt, ist ein kreuzbiederer Satz aus akademischen Stil übungen, sans genie et sans esprit ${ }^{21}$. Da möchte man einfach wünschen, Plutarch habe geschrieben, wie es die Planudeischen Kodizes wollen. Und vielleicht hat er es ja auch getan. Und handelt es sich wirklich um zwei Eingriffe, dann ist der des native speaker um eine Welt besser (zu dieser Übertreibung reizt mich Poh lenzens egregio) als der des Mannes der wissenschaftlichen Textkritik.

4. Ein letztes Beispiel aus Pohlenzens Einleitung (p. XXXIV) zeigt erneut, daß die Behauptung, D sei stark interpoliert, auf einer petitio principii beruht. Wir haben hier übrigens einen der Fälle vor uns, an denen der von Bernardakis gege bene Text nicht zu halten ist. Auf solche Passagen trifft der Benutzer der Ausgabe gelegentlich. Daß es dazu kommt, mag mit der zweifellosen Überlastung des Editors Zusammenhängen, worauf ich später noch eimal zurückkommen werde.

Bei der zu behandelnden Stelle han delt es sich um Пврi $\varepsilon v \theta v$ i $\alpha$ 471 D.

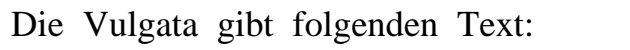

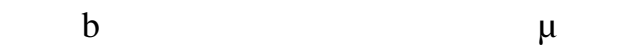

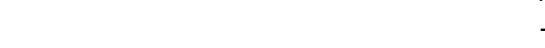

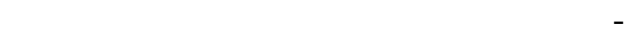
$\gamma \dot{\eta} v \alpha 1$ ह் $\dot{\alpha} \varphi O v \quad \dot{\eta} \lambda \alpha \beta \dot{\alpha} v o v \tau 1 \quad \eta \delta \dot{\varepsilon}$

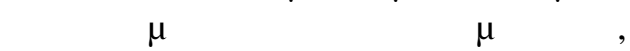

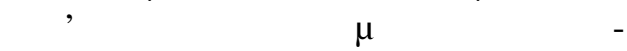

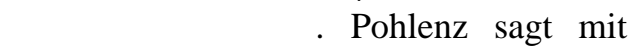
Recht, daß der Satz in Ordnung ist, wenn man mit $\mathrm{hG}^{4(!)} \dot{\hat{u}}$ statt oi schreibt. D habe nun wegen des (nach Pohlenzens Auffassung irrtümlichen) òi den Text wie folgt umgemodelt (refingit); ich hebe die Änderungen

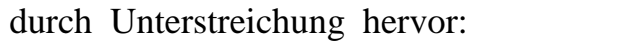

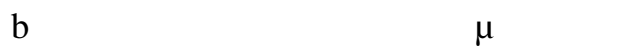

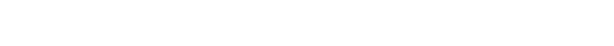

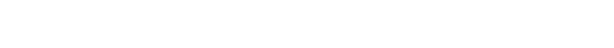

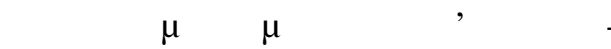

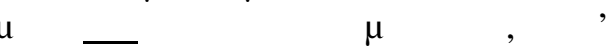
$\dot{\alpha} \beta \varepsilon \lambda \tau \varepsilon \rho i \alpha \quad \kappa \alpha i \quad \omega \rho i \alpha$ тої $\dot{\alpha} \delta v v \dot{\alpha} \tau o 1$

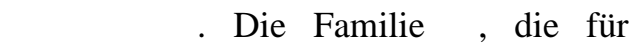
Pohlenz ja zusammen mit D die Tradition repräsentiert, habe denselben Text wie D, nur habe sie ,irrtümlich" (per errorem) das (von D hinzugefugte) oǘk ausgelassen. Der Text von $\Theta$ ist nun aber in Ordnung, und D wird, vielleicht bloß aus Unaufmerksamkeit und ver

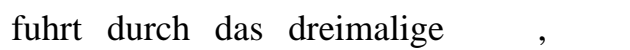
hinzugefugt haben; vielleicht hat $\mathrm{D}$ aber auch den Satz nicht verstanden und ver schlimmbessert. Der Satz lautet also, mit angemessener Interpunktion versehen:

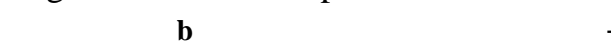

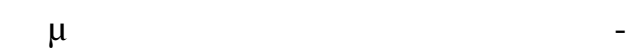

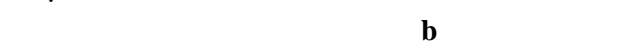

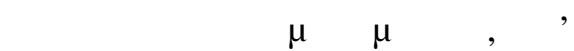

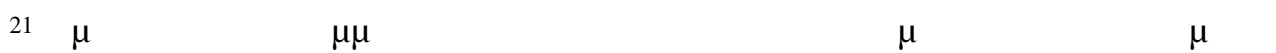

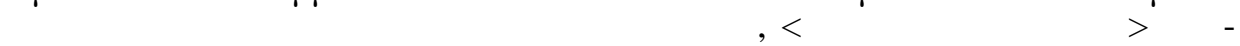

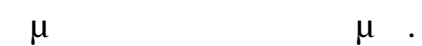




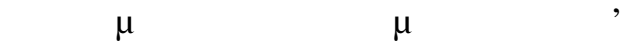

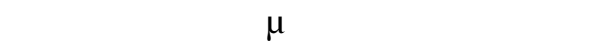

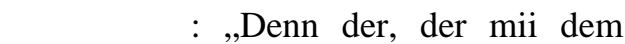
Pflug schießen und der, der mii dem Ochsen den Hasen jagen will, ist nichl unglücklich, und auch (der ist) nicht (unglücklich), der, wenn er mit Fisch netzen verschiedener Art Hirsche jagen will, sie nicht fangen kann, und solche Leute versuchen sich nicht an Dingen, denen sich ein böser Daimon widersetzt, sondern aus Albernheit und Torheit an Unmöglichem." Weder Inhalt noch Satz bau scheinen mir unplutarchisch zu sein, vor allem nicht im Zusammenhang in die ser, gelinde gesagt, stilistisch eigenwilli gen Schrift ${ }^{22}$. Das soeben Gedruckte ist allerdings nicht der Text von Bemardakis, der die Fassung von D (also mit oúk) übernimmt und im Schlußteil zum einen selbst eingreift, indem er die dativischen Substantive in den Nominativ versetzt,

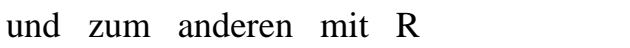
schreibt. Was so herauskommt, ist (und zwar wegen des oúк) inhaltlich nicht zu verstehen . Der Satz der Vulgata (mit der Verbesserung $\dot{u}$ ) kann sich auch sehen lassen, empfiehlt sich aber nicht in höherem Grade als derjenige von $\Theta$.

Ich will nicht sagen, daß D damit schon rehabilitiert ist und schon gar nicht, daß in die Handschrift nicht auch hineininterpoliert worden ist. So folgt

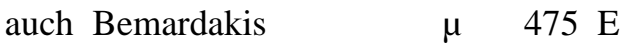
der Überlieferung bei Stobaios und zitiert im Kodex nicht einmal die Art, wie D die in allen unseren Handschriften zu findende Glosse in den Satz einbaut, sondern nur das, was er in der Vulgata vorfindet ${ }^{24}$. $\mathrm{Zu}$ einer Rehabilitiemng von D fehlen mir die Mittel. Eine solche Teilrehabilitierung ist von anderer Seite erfolgt. Inzwischen sind die Moralia nämlich prinzipiell unabhängig von Bemardakis und der zweiten Teubneriana in der Collection Bude neu ediert worden. Einer der Herausgeber ist der Editor der Biographien Plutarchs in der selben Sammlung, Robert Flaceliere, einer der herausragenden Plutarchkenner des 20. Jahrhunderts. In der Einlei tung zu seiner Ausgabe der Dialogues Pythiques (Oeuvres morales VI, Paris, 1974), stellt er fest, daß es wohl keine Hoffnung auf ein Stemma der Hand schriften gebe (also auf jene den Arche typos rekonstruierende Anordnung, die sowohl unserem Hang zum Eliminieren als auch unserer Liebe zu Autoritäten so erfreulich entgegenkommt). Da dem so sei, ordne man am besten die Handschriften nach ihrem Alter an, was aber nicht bedeute, daß die altersglei-

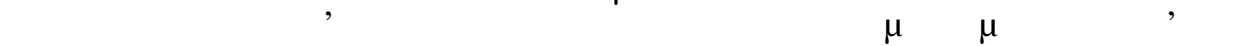

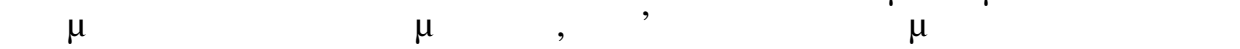

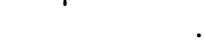

Vgl. Verf., „Sprungbrettargumente bei Plutarch“ (wird gedruckt).

Vgl. Poh lenz, $\rho$. XXXI, mit Bernardakis, zur Stelle. 
chen Handschriften eine gemeinsame Quelle hätten. Die ältesten Kodizes seien (einfach deswegen) am verläßlichsten, weil sie am wenigsten interpoliert seien. Zur ältesten Gruppe (für die Pythischen Dialoge, wo der ein Jahrhundert ältere Palimpsest ausfällt), gehört, mit nur drei anderen, der Kodex D aus dem 11. Jahrhundert. Es folgen die Gruppe aus dem 13. (sic) und 14. Jahrhundert, darun ter die Planudei, und aus dem 15. Jahr hundert. Was durch diese Einschätzung erfolgt ist, kann man mit gutem Recht den Beginn einer Relativierung der Versuche nennen, die Wahl des Kodex D als Textgrundlage zu Grundlage des Wilamowitzschen Urteils, fundamentum recensionis non esse iactum, $\mathrm{zu}$ machen, denn schließlich gehört er ja jetzt zu den verläßlichsten. Nach einer etwas späteren Ausgabe in derselben Reihe (Oeuvres morales VII 1, hg. von Jean Dumortier und Jean Defradas, Paris 1975) gibt es 3 Familien, D gehört in die zweite, weniger gute, und es wird kein Wort über diese Handschrift verloren. Im Wesentlichen haben wir hier die Erkenntnisse der neuen Teubneriana vor uns. Dieser ist übrigens durch die Bude-Ausgabe nicht der Garaus gemacht worden: Ihr erster Band wurde 1993 wieder nachgedruckt. In guten Händen scheint der Leser mit der Ausgabe von De audiendo durch Brian P. Hillyard (New York 1981) zu sein. Hillyard geht von 4 Hyparchetypoi aus; D ist die Leithandschrift eines, des 4., von ihnen, Bb. Leider kein Wort über die -Tradition, auf die Pohlenz uns so gespannt gemacht hat. Aber immerhin: D ist, alles in allem, offenbar doch nicht der unheimliche Zeuge, als den ihn uns Pohlenz noch vorstellt. Weiteren For schungen darf Bernardakis von der Insel der Seligen aus gelassen entgegensehen.

\section{unus homo}

Ich komme nun zur Besprechung der Kritik an der handwerklichen Seite der Arbeit von Gregorios Bernardakis. Da ist zunächst der Angriff auf seine Kollation, d.h. die Akkuratesse des Vergleichs der Kodizes. Ich sagte oben, daß es sich bei den Moralia um an die 80 Schriften handelt (von der Masse der Fragmente im 7. Band der Bemardakisausgabe sehe ich dabei natürlich ab). Der Vergleich von Kodizes angesichts dieser Menge ist eine Lebensaufgabe.

Bernardakis legt, in der Ausgabe selbst, Wert auf die Feststellung, daß er eine sogenannte editio minor veranstaltet, d.h. eine vorläufige Ausgabe, die noch nicht all den Ansprüchen gerecht werden kann, die an eine eigentliche, also an eine editio maior, gestellt werden müssen. Der textkritische Apparat wird kleiner sein; alles ist darauf abgestellt, dem Leser eine Lektüre zu ermöglichen, die in ihm das Interesse an mehr Wissen weckt (I, Vllf.; II, X). Die editio maior oder amplior wird von Bernardakis selbst noch ins Auge gefaßt (I, VIII, XI), kommt aber unter sei ner Herausgeberschaft nicht zustande. Das entschuldigt keine Flüchtigkeiten, weist aber auf ganz allgemeine Weise die Dimensionen auf, innerhalb deren Kritik geübt werden sollte. Auch hier ist die 
Kritik von Pohlenz verständnisvoll und gerecht. Er schreibt: „Wenn er aber die Handschriften noch (!) nicht mit der nöti gen Sorgfalt untersucht hat, so ist das selbstverständlich verzeihlich, weil er, als Einzelperson, unus homo, einer solchen Aufgabe nicht gewachsen war" ${ }^{25}$.

Die Editoren der zweiten Teubneriana ahnten es also: Sie waren an eine Arbeit gegangen, die sie eine Weile beschäftigen sollte, und deren erste Bände schon längst in zweiter Auflage erschienen waren, als die letzte Schrift ihre erste noch nicht erlebt hatte.

Pohlenz schreibt, wie schon gesagt, Wilamowitz habe die Korrekturen der Ausgabe mitgelesen (p. VI). Sind aber die soeben zitierten Worte Pohlenzens das endgültige Urteil auch von Wilamo witz, dann hat er uns von der Last befreit, ihm seine ursprüngliche, wenig differenzierte Kritik auf Dauer nachzu tragen. Was wir gehört haben, bedeutet ein Einlenken auf die vernünftigen Bahnen konstruktiver Kritik und ist weit entfernt von den Ungehörigkeiten der ersten Aufregung. Ich kann die Richtig keit des Urteils, Bernardakis habe nicht immer mit der nötigen Sorgfalt kollatio niert, nicht nachprüfen; Babbitt wird, nach eigener Recherche, die Kritik an der Bernardakisschen Kollation wieder holen. Also gehe ich davon aus, daß es stimmt, und frage mich, ob es nicht, zusätzlich zum Pohlenzschen Argument „Überforderung“, noch einen weiteren Entschuldigungsgrund gibt.

Man sollte sich nämlich fragen, wie wichtig solche Versehen für speziell diese Ausgabe sind. Und da ergibt sich doch, daß dann, wenn eine Ausgabe prinzipiell auf einer Art codex unicus beruht, wie Pohlenz das Verfahren von Bernardakis beschreibt, die Kollation der nicht als certisismi duces angesehenen Handschriften bei weitem keinen solchen Stellenwert hat wie bei einer Ausgabe, die auf einer dem Archetypos geltenden recensio basiert. Eine eilige Kollation in der Art einer gro ben Übersicht dürfte ausreichen. Dabei sind Fehler gemacht worden? Das haben eilig gemachte grobe Übersichten so an sich. Die Fehler müssen von der Kritik benannt und vom Bearbeiter oder seinen Nachfolgern verbessert werden, und damit wäre der Kritik Genüge geschehen.

\section{$<\ldots>$ a good many readings of}

Bernardakis's text have been si lently corrected ${ }^{16}$

Kommen wir also zu den Ver besserungen des Herausgebers, seinen sog. Konjekturen. Daß der Editor selbst in den Text eingreift, wo es ihm nötig erscheint, ist sein gutes Recht. Trotzdem sehe ich in diesem Punkt eine zeitbe dingte Eigentümlichkeit der Ausgabe, die auch ich lieber nicht so vorfinden würde. Zu der Zeit, als Bernardakis

25 a.a.O. XIV.

26 Plutarch's Moralia etc. (siehe Anm. 3), Band III (1929), p. VIII; vgl. Band I, p. VIIf. 
seine Ausgabe machte, war das Konjek turenmachen aber in geradezu gefährli chem Maße modern. Man bezeichnet diese Epoche gern kritisch und ironisch als die der „Konjekturalgenies“.

Das Gesamtprofil des Wissenschaft lers Bernardakis weist ihn als einen soge nannten Textphilologen aus. Er war mehr als das. So veröffentlichte er ein Autoren lexikon, das 1922 in zweiter Auflage erschien $^{27}$. Die meisten seiner Arbeiten befassen sich aber mit Verbesserungen von korrupt überlieferten Stellen, mit der Polemik gegen seiner Ansicht nach fal sche Verbesserungsvorschläge anderer Philologen oder mit der damit eng Zusammenhängen recensio. Hier kannte er keine Scheu. Bereits 1877 griff er mit einer eigenen kleinen Schrift den größten damals lebenden Textphilologen, Carl Gabriel Cobet, an, und begründete am Schluß, warum: Weil die Irrtümer bedeu tender Wissenschaftler besonders verhee rend zu wirken pflegen. Das war und ist in der ständischen Welt der Universität ein einigermaßen mutiges, aber natürlich richtiges Wort. Als alter Mann veröffent lichte er, 1918, ein über 1200seitiges „Kritisches Lexikon“, in welchem er von in alphabetischer Reihenfolge angeord neten Einzelwörtem ausging, mit deren textkritischer Behandlung er nicht zufrie den ist. Bernardakis gehört, was dieses Interesse an Texteingriffen und Dis kussionen darüber angeht, in eine Zeit, die sich in seinem eigenen Alter dem Ende zuneigte.

Akzeptieren wir also, daß viele Vor schläge von Bernardakis keine Zustimmung gefunden haben.$^{-*}$

Es kommt noch etwas hinzu, was der Angelegenheit weiter an scheinbarer Brisanz nimmt. Der textkritische Appa rat von Bernardakis ist insgesamt wohl tuend übersichtlich und die Eingriffe des Herausgebers in den Text sind, im Apparat, durch einen Asteriskus gekenn zeichnet, der angesichts der im allge meinen ganz wenigen Zeilen, die der Apparat einnimmt, sofort beim Umblättem in die Augen fallen. Der Leser braucht nicht, wie so oft in Ausgaben, den Apparat regelrecht mitzulesen, um das ihm Wichtige zu finden, sondern das

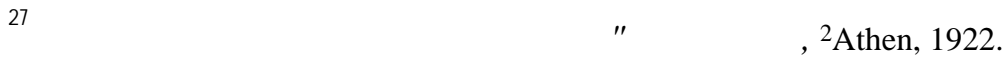

•* Indem wir die Angelegenheit auf den richtigen Grad ihrer Brisanz zurückführen wollen, bekommen wir Schützenhilfe von der wünschenswertesten Stelle: Paul Ma as. S. 13 sei ner Textkritik stehen die goldenen, in einem erfrischenden Paukenschlag gipfelnden Worte: „Daß die Konjekturalkritik eine Zeitlang grundsätzlich bekämpft wurde [geschrieben 1927 oder vorher!!, sei als vorübergehende Verirrung der Forschung nur eben erwähnt. Natürlich ist es viel schädlicher, wenn eine Verderbnis unerkannt bleibt, als wenn ein heiler Text zu Unrecht angegriffen wird. Denn jede Konjektur reizt zur Widerlegung, durch die das Verständnis der Stelle jedenfalls gefördert wird, und nur die besten werden sich durchsetzen $\langle\ldots$... Wer sich fürchtet, einen unsicheren Text zu geben, wird besser tun, sich nur mit Autographa zu beschäftigen.“
} 
Wichtigste, die von der Überlieferung ab weichenden Vorschläge des Herausge bers, bekommt er von Bemardakis ge wissermaßen als Blickfang präsentiert. Es hindert ihn nichts, alles, was er will, bei der Lektüre rückgängig zu machen. Ab gesehen davon sind viele Eingriffe selbst verständlich richtig oder bedenkenswert, und manches hat sich auch in spätere kon kurrierende Ausgaben gerettet.

\section{Exemplarisches und Ausblick.}

Habe ich bisher in der Hauptsache gefragt, was an der Plutarchausgabe von Bernardakis verteidigt werden kann, so resümiere ich nun, was ich an ihr gut und beispielhaft finde.

Die Einleitung zum ersten Band dieser Ausgabe beschreibt die verwendeten Kodizes und setzt sich mit den Er gebnissen von Max Treu auseinander, der damals der führende Forscher auf dem Gebiet der Überlieferung der Moralia war. Das ist Usus: Es gehört in jede Praefatio einer Ausgabe. Das, was auf die Be schreibung der Kodizes bei Bemardakis folgt, sollte man nun aber mit editorum in usum (als Beispiel für spätere Herausgeber) überschreiben . Bernardakis führt den Benutzer seiner Ausgabe in den Stil Plutarchs ein. Natürlich ist die Ein schätzung des Stils eines Autors das Fundament für die Auswahl der Überlie ferung, der man folgt (und nicht die Rekonstruktion einer Überlieferungslinie, auf der, wie im Fall Plutarchs, unsere Kenntnis aus nicht mehr als bloßem Zufall beruhen kann). Erst recht ist sie das Fundament für die Korrektur dieser Über lieferung und ihres besten Zeugen. Nach dieser Einführung kann der Leser ein Wörtchen mehr mitreden, als wenn er lediglich ein hierarchisches System von Textzeugen präsentiert bekommen hat. Eben diese Auffassung vertritt Bemarda kis, editorum in usum, ausführlich, indem er sich die Mühe macht, an vielen Stellen, wenigstens prinzipell, zu zeigen, wie die Emendation des Textes von der Kenntnis des Stils des Autors geprägt sein muß.

Ich muß bei diesem Thema noch einen Moment verweilen.

Meine soeben vorgetragene These, daß nicht die recensio, also die Ordnung der Kodizes nach Zuverlässigkeit und gegenseitiger Abhängigkeit, sondern die Kenntnis des Stil eines Autors (und seine Aneignung durch den Editor) am Anfang der constitutio textus steht, kann leicht angegriffen werden. Man muß ja nur fragen, wie man denn den Stil eines Autors kennenlernen will, wenn man nicht vorher einen sicheren Text hat. Also müsse man zunächst diesen herstellen, und das gehe nicht ohne recensio (also nicht ohne den Teil der Arbeit, den Bernardakis nicht vorgelegt haben soll). Die Rückfrage, wie ich denn überhaupt während der recensio u.a. Fehler, insbeOxford, 1945. 
sondere Stilfehler feststellen will, um die Abhängigkeit der Kodizes zu bele gen, wenn ich dazu zuerst meine recen sio fertiggestellt haben muß, führt dann $\mathrm{zu}$ einem Waffenstillstand, der den in der Geisteswissenschaft immer wieder un ausweichlichen circulus in interpretan do auch für diesen Fall bemüht. Wer auf der recensio als erstem Schritt behairt, wird das tun, weil er eine, wie er meint, „objektive“ Grundlage für alles weitere, also auch die Aussagen über den Stil des Autors, braucht, weil er ,wissenschaft lich“ Vorgehen will. Es ist schon deutlich geworden, wie ich zu dieser Art Wissenschaftlichkeit stehe. Schon wäh rend der recensio, erst recht an ihrem Ende, wenn es um die emendatio textus geht, hat der „Wissenschaftler“ immer wieder mit den Instrument seiner ganz per sönlichen Sensibilität zu tun. Wenn ihm die abgeht, kommt er gar nicht zu Kriterien, die ihn im Einzelfall entschei den lassen, ob dieser Text oder jener dem Autor ,zuzutrauen“ ist. Und als Feld' auf dem diese Sensibilität zur Wirkung kom men kann, ist eine bis ins letzte ausgefeilte Ausgabe, somit auch eine exakte recensio, nicht nötig. Der Stil eines Autors ist auch aus einem noch unvollkommen edierten Text, sogar aus einem fehlerhaften Kodex zu erfassen, wenn dieser nicht völlig aus dem Rahmen des Üblichen fällt.

Auf dieser frühen Ebene ist Textkritik

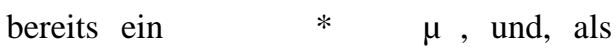
solches, nicht eine Angelegenheit exakter Wissenschaft, sondern von $\sigma \tau \varnothing \chi \alpha \sigma$ ò .
Der Blick, das Auge, die Fähigkeit, sich in einen Stil „einzuleben“, lauter Fähigkeiten, die keineswegs irrational sind, für die aber oft keine andere Rechenschaft möglich ist als die Meister schaft dessen, der uns einen Lösungsweg vorschlägt, sie stehen am Anfang der Arbeit, sind ihre Bedingung und ihr wesentliches Kriterium. Die beste Konjektur ist nicht die, die sich auf eine Juristen und Schulmeistern abgesehene Methode der Rechtfertigung von $\mathrm{Ab}$ hängigkeitszuweisungen und oft genug auf die sprachlichen Ideale neuzeitlicher Stilübungen stützt, nicht die, die von dem erwarteten Konsens der denselben Methoden folgenden Kollegen begrüßt wird und mir ein gewisses $\mathrm{Ma} ß$ an Risikofreiheit in der Welt des Gnomon sichert, sondern die, die dem Autor, im Rahmen seines Niveaus, am meisten ent spricht. Der Plutarcheditor muß ein begabter Textrestaurateur und ein $\Pi \lambda \mathrm{ov}$ $\tau \alpha \rho \chi 1 \kappa o \dot{~ s e i n . ~ D e s w e g e n ~ w a r ~ e s ~ n i c h t ~}$ nur richtig, sondern weise, den Leser in den Stil des edierten Autors einzuführen und eo ipso die Kriterien des Editors klarzumachen, auf Grund deren recensio und emendatio versucht wird. Das Vor gehen von Bemardakis wird zwar in Einzelfällen immer wieder einmal ange wandt, hat aber, aufs Ganze gesehen, keine Schule gemacht. So bleibt seine Ausgabe in diesem Punkte beispielhaft.

Ich komme nun zum vielleicht kon troversesten Punkt meiner Ausführun gen. Ich bin der Meinung, daß man die Ausgabe von Bemardakis nicht als über- 
holt abtun sollte ${ }^{30}$. Die Kollationen, die zur Vorbereitung der zweiten Teubneriana veranstaltet worden sind, haben nolentes volentes den Verdacht genährt, daß die -Tradition in besondere Nähe des Autographs führt. Man bleibt neu gierig. Wenn nun zusätzlich die Tendenz neuerer Ausgaben dahin geht, die Hand schrift D aus der Aschenputtelecke her auszuholen, in die Pohlenz sie noch gesteckt hat, so hält dieser Umstand wenigstens die Neugier lebendig. Wenn also die Bernardakisausgabe eine Edition ist, der mit D die Leithandschrift einer der wichtigsten Handschriftenfa milien zugrundeliegen sollte, und ferner eine Edition, der ein gewisser Respekt vor Planudeischem Textmaterial nachge sagt werden kann, so steckt darin der Anreiz zu weiteren Nachforschungen. Man vergleiche das Ergebnis der more Bernardacis zustandekommenden Text konstitution mit anderen Textkonstitutio nen. Man lernt auf jeden Fall mehr dabei, als wenn man beim bloßen Erwägen einer der Lesarten von D schon auf eine Abwehrhaltung verfällt: Aber genau dahin kann uns Pohlenzens gewundene gegen D gerichtete Argumentation brin gen. Bernardakis also nicht aus den Augen befördern, heißt das, sondern immer wieder einmal hineinsehen. Was mich angeht, so habe ich meinen Plutarch in dieser Ausgabe gefunden. Der Band III der Bernardakisausgabe liegt meiner 1970 fertiggestellten Habilitationsschrift zugrunde. Die oben S. 118f. zitierten griechischen Worte, also die

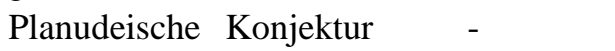
531 Ff., sind wörtlich und ohne Diskussion von mir übernommen ${ }^{31}$. Bisher hat mir noch niemand die Wahl meiner Ausgabe vorgeworfen, obwohl sie immer wieder einmal auffällt. Entweder hat man sie mir hingehen lassen, was zei gen würde, daß die Bernardakisausgabe als Altemativausgabe schon akzeptiert ist, oder man hat nichts gemerkt. Auch daraus könnte man seine Schlüsse ziehen.

Zeichnet sich nun hier eine Aussicht $\mathrm{ab}$, an der man sich freuen kann, so will ich zuletzt auf ein besonderes Verdienst der Bernardakisschen Ausgabe kom men, eines, das wenigstens mich ihr, so wie sie ist, immer besonders gewogen gemacht hat. Das ist die darin zutagetre tende Bescheidenheit, also das Wissen um unsere Unzulänglichkeit, wenn es darum geht, nach 2000 Jahren einen so vielschichtigen und komplizierten Autor wie Plutarch zu edieren, obwohl man Sprache und Stil des Autors virtuos und wie selbstverständlich $\mathrm{zu}$ beherrschen scheint. Die zu vielen Konjekturen, die ja an sich auf starkes Selbstbewußtsein des Editors schließen lassen, werden begleitet von einer erstaunlichen $\mathrm{Zu}$ rückhaltung, die man in den meisten anderen Ausgaben vermißt. Sie zeigt sich an drei Zügen: dem malim, mit dem der Herausgeber zu erkennen gibt, daß er den Text gern anders hätte, als er ihn Meinung, l.c. XIV. 
gibt, aber trotzdem nicht wagen will, wirklich eine Änderung vorzuschlagen, und dem zweifelnden Fragezeichen, das im wesentlichen dasselbe besagt. Die gewissermaßen als Selbstanzeige zu wertende Herausstellung eigener Kon jekturen durch den Asteriskus gehört auch in dies Bild. Mir ist wohler bei einem Apparat, dessen Haltung mehr meisterliche Vorsicht zeigt, als bei der scheinbaren Selbstsicherheit anderer Apparate, die den Eindruck der End gültigkeit erwecken und sich und dem Leser damit etwas vormachen. Einige von ihnen sind extrem ausführlich und erschlagen den Benutzer mit gelehrtem Material. Andere sind von apodiktischer Kürze und wollen wohl die numinosen Sprüchen geltende Ehrfurcht erwecken.
Alles in allem glaube ich sagen zu dür fen, daß wir uns schaden, wenn wir diese Ausgabe für überholt oder endgültig ersetzt erklären. Sie sollte uns teuer und zur Ver fügung bleiben und nicht unter dem bei Altphilologen gelegentlich feststellbaren, wie Überkompensation wirkenden Moder nitätswahn zu leiden haben, wonach man unbedingt die neueste Ausgabe kennen und erst recht „benutzen“ muß. Wie Interpreta tionen aus älteren Tagen hier und da, nach Prüfung, unentbehrlich sind, so auch einige Ausgaben aus früherer Zeit, wie zum Bei spiel die hier besprochene, und zwar gera de weil sie das fundamentum recensionis so und nicht anders gelegt hat. Wenn ich damit inzwischen schon wieder offene Türen einlaufe, umso besser.

31 Plutarchs Schriften über die Heilung der Seele, Göttingen, 1971 (Hypomnemata, Band 34), S. 58 oben. 